\title{
Effect Of Portal Hypertensive Gastropathy On Electrogastrographic changes \&Gastric Emptying Time
}

\author{
*El Bokl ,Mohammed. **Hanan M.. M .Badawy , ***E 1 Maltawy, \\ Mohammed A.F,Abdul Mageed, Khalid H**.\&Ghali,Sameh M. \\ *Professor of internal medicine Ain Shams uni. \\ **Lecturer of internal medicine Ain Shams uni. \\ ***Assistant professor of internal medicine Ain Shams uni.
}

\begin{abstract}
:
Background: Dyspepsia is a frequent symptom in cirrhotic patients . Congestive gastropathy or portal hypertensive gastropathy (PHG) was detected as a possible cause of sustained dyspepsia in $40 \%$ of these patients , and prolonged gastric emptying time(GE) with decrease gastric wall compliance were detected in cirrhoric patients .

Electrogastrography (EGG) is a non-invasive method to study gastric myoelectrical activity from the body surface by electrodes . whereas, an ultrasonographic method can be used for the estimation of gastric emptying by measuring what so called half emptying and full emptying times with other parameters.

Aim of this work is to study patterns of EGG \& ultrasnographic GE. In cirrhotic patients with or without PHG . 45 patients were chosen for this study and were divided into 3 groups ; group 1 is cirrhotic patients with PHG negative upper gastrointestinal ( U.G.I.T.) endoscopy \& group 2 is cirrhotic patients with PHG positive $\&$ group 3 is healthy subjects taken as control .
\end{abstract}

Results :- we found significant delay in GE in gp. 2 in relation to group 3 (p $<0.05$ ). EGG reveiled power ratio lower in gp. 1, 2 ( $2.01-1.93$ ) respectively compared to group. 3 (2.63). Also there was highly significint increase in "Dominant frequency" at rest ( DF ) in group. 1 campared to group. $2 \& 3$ ( p>0.01) where mean of group 1 was 2944.9 , in group 2 was 2477 and in group 3 was 1934 \& the power meal at DF was higher in group 1 than 2, 3 but siatistically insignificant where the mean in group 1 was $5922.5 \&$ in group. 2 was 4804.8 and was 5087.8 in group $3(p>0.05)$

conclusion :- Delayed gastric emptying by U.S, and changes in EGG records of cirrohotic patients especially in presence of portal hypertensive gastropathy may explain dyspepsia frequently occurring with portal hypertensive gastropathy.

\section{Introduction}

Dyspepsia is a very frequent symptom in cirrhotic patients where congestive gastropathy or portal

hypertensive gastropathy (PHG) was detected as a cause of sustained dyspepsia in $40 \%$ of these patients 
(Grassi et al., 2001). And prolonged gastric emptying (GE) with decrease gastric wall compliance were detected in cirrhotic patients ( Isobe et al., 1994). Portal hypertensive gastropathy defines a wide spectrum of diffuse macroscopic lesions that appear in the gastric mucosa of patients with portal hypertension.

Gastric mucosal lesions are important cause of upper gastrointestinal bleeding in patients with portal hypertension. These gastric lesions, also called portal hypertensive gastropathy (PHG), are well established clinical entity (Smart and Triger, 1991). Congestion seems to be the underlying mechanism for the development of PHG (Groszmann and Colombato, 1988). The gross changes in portal hypertensive gastric mucosa are ascribed to possible abnormalities in the microvasculature of the portal hypertensive stomach (Smart and Triger, 1991). PHG is predominantly located in the fundus (Sarin et al., 1992) and associated with fundic gland atrophy (Nishida et al.,1989). It has been suggested that the chief cell mass is decreased in cirrhotic patients with portal hypertension. PHG has been proposed within the last 10 years to group the previously termed (haemorrhagic gastritis or diffuse gastric lesions) in patients with cirrhosis. This was the result of several studies demonstrating that dilated vessels in the mucosa, and not erosions or inflammation, are the histologic hallmark of endoscopic diffuse gastric lesions in patients with portal hypertension. The term "congestive gastropathy", which has also been used alternatively to PHG, is not fully accurate since venous congestion seems not to be the only pathogenic mechanism involved in development of such gastric lesions (Josep and Pique, 1997).
Endoscopically, PHG includes several mucosal lesions which have been classified as mild or severe ( Hashizume and Sugimachi,1995).

Mild: Mosaic pink in center, fine red speckling, scarlatina, snake skin pattern. are highly prevalent $65-90 \%$, Severe: Red spots, brown spots, diffuse haemorrhagic lesions in only 10-25\%( Josep and Pique 1997).

The mosaic pattern is defined by a white reticular network separating areas of raised red or pink mucosa resembling a snake skin. This is the most common gastric mucosal alteration in patients with portal hypertension and is predominantly found in the corpus and in the fundus. A mosaic pattern is not a specific lesion for portal hypertension unless pink or red oedematous mucosa is present in the center of the white reticula. Other mucosal lesions included in mild PHG are superficial reddening on the surface of the rugae and a fine pink speckling or "scarlitina"type rash (Josep and Pique, 1997).

The severe endoscopic lesion of PHG is characterized by discrete cherry red spots, which may progress to confluent areas of diffuse bleeding. These red spots may appear in any part of gastric mucosa, including fundus, corpus and antrum (Payen et al.,1995). Another different endoscopic finding reflecting an underlying vasculopathy is that of red strips in the gastric antrum converging on the pyloric area. This macroscopic lesion, which has been named "watermelon stomach", seen not only in cirrhotic patients but also in other diseases, mainly autoimmune or connective tissue disorders such as scleroderma, sclerodactly, hypothyr oidism, pernicious anaemia, or primary biliary cirrhosis (Gostout et al.,1992).

The unique feature of PHG on 


\section{El Bokl ,Mohammed, et al}

histologic examination is marked dilatation of the capillaries and collecting venules in the gastric mucosa. In addition submucosal veins appear ectatic, irregular and with areas of intimal thickening. When the red spots are located in the antrum, fibromuscular hyperplasia, fibrohyalinosis and thrombi are usually encountered in histological examination (Josep and Pique, 1997).

The parietal cell count in the gastric mucosa of rats with experimental portal hypertension was found to be significantly diminished (Agnihotri et al.,1996).

The prominent muscularisation of the veins was the main histological feature supporting a late stage congestive gastropathy rather than angiodysplasia (Leone et al.,1997).

The diagnosis of PHG is usually endoscopic, although it may be difficult some times to endoscopically distinguish such lesions from other gastric disorders not related to portal hypertension (Corbishley et al.,1988). Routine endoscopic biopsies obtained by conventional forceps are often unhelpful to diagnose $\mathrm{PHG}$, since from specimen obtained by such device is difficult to ascertain the presence or absence of vessel dilatation because of the patchy nature of the alteration. Endoscopic ultrasonography may detect a characteristic thickening of the gastric wall reflecting the oedema usually present in the gastric mucosa and submucosa of patients with PHG (Josep and Pique,1997). Aim of the work : is to study patterns of EGG \& ultrasonografic GE in cirrhotic patients with or without PHG.

\section{Patients And Methods}

Thirty subjects with liver cirrhosis from the internal medicine department of Ain Shams Hospital, were enrolled in this study with 15 sex and age matched control. They were classified into 3 groups as the following:

- Group I: fifteen cirrhotic patients without congestive portal gastropathy.

- Group II: fifteen cirrhotic patients having congestive portal gastropathy.

- Group III: fifteen control subjects.

The cirrhotic patients were classified according to Child Pugh classification into Child A, B and C.

\section{Exclusion criteria:}

We have excluded subjects with previous history of some diseases that might affect gastrointestinal motility as patients with diabetes mellitus, renal impairment, calcular cholecystitis, chronic obstructive airway diseases, drugs affecting gastric motility (e.g. prokinetics, erythromycin). All patients and controls were subjected to the following:

\section{1- Full history taking\&clinical examination:}

All subjects were asked espicialy about dyspeptic symptoms (e.g. nausea, vomiting, fullness, bloating, epigastric pain and reflux).

\section{2-Complete blood count.}

3- Liver function tests: ALT, AST, total and direct bilirubin, s. albumin and prothrombin time.

\section{4-Fasting blood sugar and kidney functions tests.}

5-Upper gastrointestinal endoscopy:

The endoscopy was done in Ain Shams Hospital in the endoscopy theater by the use of Olympus CIF Type 2 T 200 vidioscope gastroduoden oscope.

The patients were fasting for at least 6 hours and the endoscopic examination was done to detect the presence or absence of esophageal or 
fundal varices, and the presence of congestive portal gastropathy and patients were divided into two groups:Mild ( Mosaic pink in the center, fine red specling \&snake skin pattem) 2 - severe (red spots \& brown spots).

6-Abdominal ultrasonography for assessment of gastric emptying:

All subjects were fasting for at least 6 hours before examination.

Abdominal U/S was performed with 3.5 array transducer.

The following markers of gastric motility were measured:

a-Basal antral area: was the mean of two measurements taken 0 and $5 \mathrm{~min}$. before the meal.

b-Maximal postprandial antral area: was measured after maximal widening of the antrum had occurred, usually within 2 min. postprandially.

c-Minimal postprandial antral area: was the smallest area measured at any time postprandially.

d-Half emptying time: was the time in minutes to observe a $50 \%$ decrease in maximal antral area ( $\mathrm{t} 1 / 2$ time). Calculated by linear regression analysis from the linear part of antral emptying curve. Antral emptying curves were obtained by plotting antral area versus time.

The semisolid test meal (11g fat, $120 \mathrm{Kcal}$ in the form of butter was emulsified in $200 \mathrm{ml}$ of tap water) swallowed at room temperature over a one min. period .

\section{7-Elecrogastrography:}

Gastric electrical activity was recorded from five disposal pregelled silver/silver chloride surface electrodes placed on the upper abdomen. This was done after the skin has been carefully abraded to decrease resistance to obtain a good signal to noise ratio (Chen et al., 1994)

The patient was kept in a reclining position to minimize motion artifacts. Four EGG signals were recorded bipolary from these 5 electrodes as the potential differences between each of the four electrodes, and one central electrode. A reference electrode was placed at the left clavicle.

The EGG signal is polluted by signals from extragastric sources. One of these is respiration artifact, other signals considered as noise in the EGG signals; electrode potential variation (electrode noise), motion artifacts, potential variations produced by other internal organs containing smooth muscles. The electrical signals are recorded with appropriate amplification and filtering.

One hour recording while the patient is fasting was done, then given a standardized test meal (pastes and $250 \mathrm{ml}$ milk) and postprandial recording for one hour was done (Parkman et al.,1995).

After the recording session The EGG signal were subjected to spectral analysis (Fast Fourier Transform).

The mean of the power spectra for the entire recording period was calculated. The EGG signal, the highest power in the $3 \mathrm{cpm}$ band, was then selected for further analysis. The mean frequency of the normal $3 \mathrm{cpm}$ component, and its standard deviation and its power content was calculated for the fasting and postprandial period. Higher harmonics were identified in the spectrum using the criteria that they occur at frequencies that are exact multiples of the fundamental frequency, and that their power should be at least $5 \%$ of the power of the fundamental component. The early postprandial frequency dip of the normal $3 \mathrm{cpm}$ gastric component was identified. The frequency minimum and the subsequent frequency maximum of the dip were calculated by means of line to line analysis of the first 10 running spectra 


\section{El Bokl ,Mohammed, et al}

after the meal. The power ratio (the ratio of the power of the mean spectrum of the postprandial state to the power of the mean spectrum of the fasting state), is indicative of the postprandial increase in gastric motor activity and was calculated for the first hour of the postprandial period.

Dysrhythmia was defined as follows: A tachygastria was considered to be present when the power spectrum contained a sharp-peaked component with a frequency $>3.7 \mathrm{cpm}$ and $<10.8$ cpm, which was not of respiratory origin. For a definite diagnosis of tachygastria it was required that at the same time the normal gastric signal (2.6-3.7 cpm) was absent in all four EGG signals and that the abnormal rhythm was present for at least $2 \mathrm{~min}$.

A so-called bradygastria was defined as presence of a sharp peak at a frequency less than $2.6 \mathrm{cpm}$, in the absence of a normal $3 \mathrm{cpm}$ component in all four EGG leads (Jebbink et al., 1995).

\section{Statistical methodology:}

SPSS- windows- version (8) was used for analysis of this data as follows: Description of quantitative variables in the form of means, standard- deviation and range.

Description of qualitative variables in the form of frequency and percentages.

Correlation coefficient test ( $r$ test) was used to rank different variables against each others either directly or indirectly.

One way ANOVA test was used to compare more than two groups as regards quantitative variables.

Chi-square test used to compare qualitative variables.

\section{Significant level $(P)$ value:}

P> 0.05= insignificant test.

$\mathrm{P}<0.05=$ significant test.

$\mathrm{P}<\quad 0.01=$ highly significant test.

\section{RESULTS}

Table (1): Age distribution among the studied groups.

\begin{tabular}{|l|l|l|l|l|l|}
\hline Group & GroupI & GroupII & GroupIII & t value & P \\
\hline Age & $50.4 \pm 15.2$ & $50.8 \pm 9.7$ & $49.6 \pm 17.2$ & 0.3 & $>0.05$ \\
\hline
\end{tabular}

$\mathrm{P}>0.05=$ insignificant .

The mean age of group I (patients without portal gastropathy) was 50.4, the mean age of group II (patients with portal gastropathy) was 50.8 , while that of group III (controls) was 49.6; with an insignificant difference between the three groups.
In group I, $80 \%$ were males \& $20 \%$ were females; in group II, $80 \%$ were males \& $20 \%$ were females; while in group III, $60 \%$ were males \& $40 \%$ females without statistically significant difference.

Table (2): Gender distribution among the study groups.

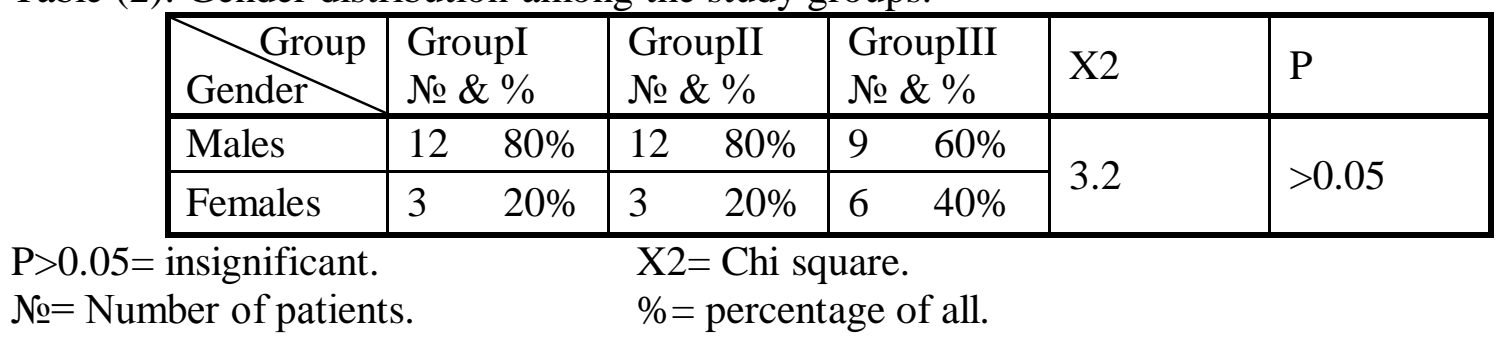


Patients of group I\&II, were divided as

following: $46.7 \%$ of Child A, $33.3 \%$ regards Child Pugh classification as the

Child B and 20\% Child C group.

Results of EGG parameters in different study groups are shown in table (3):

Table (3): Comparison of the three groups as regards the mean of results of EGG:

\begin{tabular}{|c|c|c|c|c|c|}
\hline EGG Group & GroupI & GroupII & GroupIII & t value & P \\
\hline \%CPM rest & $69.4 \pm 27.5$ & $66.7 \pm 38.2$ & $73.85 \pm 20.8$ & 0.24 & $>0.05$ \\
\hline \%CPM meal & $68.58 \pm 30.6$ & $63.4 \pm 36.13$ & $73.8 \pm 25.07$ & 0.3 & $>0.05$ \\
\hline DF & $3.03 \pm 4.64$ & $2.6 \pm 0.82$ & $2.99 \pm 0.4$ & 0.35 & $>0.05$ \\
\hline \%power rest & $19.65 \pm 7.06$ & $16.7 \pm 5.5$ & $16.6 \pm 3.4$ & 1.2 & $>0.05$ \\
\hline $\begin{array}{c}\text { \%power } \\
\text { meal }\end{array}$ & $23.6 \pm 11.6$ & $23.5 \pm 8.9$ & $22.06 \pm 6.14$ & 0.6 & $>0.05$ \\
\hline $\begin{array}{c}\text { Power rest } \\
\text { DF }\end{array}$ & $\begin{array}{c}2944.9 * \\
\pm 2814.4\end{array}$ & $\begin{array}{c}2477.13 \\
\pm 2640.3\end{array}$ & $\begin{array}{c}1934 * \\
\pm 874.7\end{array}$ & 5.2 & $<0.01$ \\
\hline $\begin{array}{c}\text { Power meal } \\
\text { DF }\end{array}$ & $\begin{array}{c}5922.5 \\
\pm 4201.8\end{array}$ & $\begin{array}{c}4804.8 \\
\pm 2967.8\end{array}$ & $\begin{array}{c}5087.8 \\
\pm 2095.8\end{array}$ & 1.9 & $>0.05$ \\
\hline PR & $2.01 \pm 1.24$ & $1.93 \pm 1.76$ & $2.63 \pm 1.57$ & 0.76 & $>0.05$ \\
\hline
\end{tabular}

$\mathrm{P}>0.05=$ insignificant.

$\mathrm{P}<0.05=$ significant

$\mathrm{P}<0.01=$ highly significant .

The mean of \% CPM at rest (CPM: cycle per min.) was 69.4 in group I , 66.7 in group II and 73.9 in group III (controls), which means that there is difference between the three groups but this was statistically insignificant $(\mathrm{P}>0.05)$.

On studying dyspeptic symptoms among the 3 groups we've found that: $70 \%$ of patients were complaining of
$\mathrm{CPM}=$ cycle per minute.

$\mathrm{PR}=$ power ratio.

$\mathrm{DF}=$ dominant frequency.

Table (4):Relation between dyspeptic symptoms and EGG parameters:

\begin{tabular}{|c|c|c|c|c|}
\hline $\begin{array}{c}\text { Symptoms } \\
\text { EGG }\end{array}$ & Present & Absent & $\mathrm{t}$ & $\mathrm{P}$ \\
\hline \%CPM rest & $68.9 \pm 34.6$ & $65.8 \pm 29.1$ & 0.23 & $>0.05$ \\
\hline \%CPM meal & $64.5 \pm 32.3$ & $69.8 \pm 36.9$ & -0.38 & $>0.05$ \\
\hline \%power rest & $17.7 \pm 5.5$ & $14.5 \pm 8.6$ & -0.67 & $>0.05$ \\
\hline \%power meal & $24.6 \pm 9.3$ & $20.5 \pm 11.8$ & 0.98 & $>0.05$ \\
\hline DF & $2.7 \pm 0.7$ & $3.1 \pm 0.6$ & 1.4 & $>0.05$ \\
\hline Power rest DF & $2540.5 \pm 2604$. & $3179.8 \pm 3055.7$ & -0.57 & $>0.05$ \\
\hline Power meal DF & $5338.45 \pm 3864$ & $5433 \pm 3074.2$ & -0.06 & $>0.05$ \\
\hline PR & $2.89 \pm 1.6$ & $2.38 \pm 1.2$ & 0.8 & $>0.05$ \\
\hline
\end{tabular}

Results of EGG study in different group were as follows:- 


\section{El Bokl ,Mohammed, et al}

The mean of \%CPM at meal was 68.58 among group I, 63.4 in group II and 73.8 in group III with an insignificant difference between the studied groups $(\mathrm{P}>0.05)$.

The mean of DF (DF: Dominant frequency) in group I was 3.05, 2.6 in group II and 2.99 among group III with an insignificant difference $(\mathrm{P}>0.05)$.

The mean of $\%$ of power at rest was 19.65 in group I, 16.7 in group II and 16.6 in group III with statistically insignificant difference $(\mathrm{P}>0.05)$.

The mean of $\%$ of power at meal among group I was 23.6, 23.5 in group II and 22.06 in group III with no statistically significant difference $(\mathrm{P}>0.05)$.
The mean of power at rest of DF was 2944.9 in group I, 2477 among group II and 1934 in group III, with highly significant difference $(\mathrm{P}<0.01)$.

The mean of power at meal of DF was 5922.5 in group I, 4804.8 in group II, and 5087.8 in group III, with statistically insignificant difference $(\mathrm{P}>0.05)$.

The mean of power ratio (PR) was 2.01 in group I, 1.93 in group II and 2.63 in group III, so the PR was lower in patients with congestive portal gastropathy compared to group I and III, but without statistically significant difference $(\mathrm{P}>0.05)$.

\section{Results of EGG parameters in correlation with grades of Child Pugh classification; This relation is shown in table (5):}

Table (5): Difference between grades of Child pugh classification as regards EGG parameters:

\begin{tabular}{|c|c|c|c|c|c|}
\hline $\begin{array}{c}\text { Group } \\
\text { EGG }\end{array}$ & Child A & Child B & Child C & $\mathrm{T}$ & $\mathrm{P}$ \\
\hline$\%$ CPM rest & $77.8 \pm 24.8$ & $54.07 \pm 35.7$ & $68.8 \pm 40.8$ & 1.6 & $>0.05$ \\
\hline $\begin{array}{c}\% \text { CPM } \\
\text { meal }\end{array}$ & $79.9 * \pm 22.3$ & $\begin{array}{c}47.8 \\
* \pm 36.98\end{array}$ & $63.8 \pm 36.9$ & 3.19 & $<0.05$ \\
\hline \%power rest & $21.4 * \pm 5.9$ & $14.3 * \pm 5.5$ & $17.13 \pm 5.6$ & 4.6 & $<0.05$ \\
\hline $\begin{array}{c}\text { \%power } \\
\text { meal }\end{array}$ & $26.1 \pm 10.5$ & $18.7 \pm 6.7$ & $25.4 \pm 11.9$ & 1.8 & $>0.05$ \\
\hline $\begin{array}{c}\text { Power rest } \\
\text { DF }\end{array}$ & $\begin{array}{c}3310.9 \\
\pm 2729\end{array}$ & $\begin{array}{c}2100.7 \\
\pm 2878.9\end{array}$ & $\begin{array}{c}2328.5 \\
\pm 2413.3\end{array}$ & 0.65 & $>0.05$ \\
\hline $\begin{array}{c}\text { Power meal } \\
\text { DF }\end{array}$ & $\begin{array}{c}6418.2 \\
\pm 3990.4\end{array}$ & $\begin{array}{c}4698 \\
\pm 3585.7\end{array}$ & $\begin{array}{c}4012.5 \\
\pm 2286.7\end{array}$ & 1.19 & $>0.05$ \\
\hline PR & $2.4 \pm 1.04$ & $3.17 \pm 1.7$ & $2.79 \pm 2.07$ & 0.62 & $>0.05$ \\
\hline
\end{tabular}

$\mathrm{CPM}=$ cycle per minute. $\quad \mathrm{DF}=$ dominant frequency.

$\mathrm{P}>0.05=$ insignificant. $\quad \mathrm{PR}=$ power ratio.

$\mathrm{P}<0.05=$ significant.

There was a significant increase in $\% \mathrm{CPM}$ at meal among Child A compared to Child B group $(\mathrm{P}<0.05)$.
There was a significant increase in $\%$ power at rest among Child A compared to Child B group $(\mathrm{P}<0.05)$. 
The effect of gender on EGG parameters among cases and controls is shown in tables (6) \&(7):

Table (6): Effect of gender among cases on EGG parameters.

\begin{tabular}{|c|c|c|c|c|}
\hline EGG Gender & Male & Female & t value & $\mathrm{P}$ \\
\hline \%CPM rest & $68.5 \pm 32.7$ & $66.6 \pm 36.2$ & 0.12 & $>0.05$ \\
\hline \%CPM meal & $67.2 \pm 30.9$ & $61.3 \pm 43.5$ & 0.3 & $>0.05$ \\
\hline DF & $2.8 \pm 0.6$ & $2.8 \pm 0.97$ & 0 & $>0.05$ \\
\hline \%Power rest & $18.3 \pm 6.9$ & $17.9 \pm 4.3$ & 0.13 & $>0.05$ \\
\hline \%power meal & $238 \pm .10 .6$ & $22.3 \pm 7.8$ & 0.33 & $>0.05$ \\
\hline Power rest DF & $2485 \pm 2576$ & $3614 \pm 3204$ & -0.9 & $>0.05$ \\
\hline Power meal DF & $5304.5 \pm 3835$ & $5600 \pm 2867$ & -0.18 & $>0.05$ \\
\hline PR & $2.8 \pm 1.5$ & $2.38 \pm 1.4$ & 0.67 & $>0.05$ \\
\hline
\end{tabular}

$>0.05=$ insignificant.

$\mathrm{CPM}=$ cycle per minute.

$\mathrm{PR}=$ power ratio.

$\mathrm{DF}=$ dominant frequency.

Table (7): Effect of gender among controls on EGG parameters:

\begin{tabular}{|c|c|c|c|c|}
\hline EGG Gender & Male & Female & T & P \\
\hline \%Power rest & $16.4 \pm 3.6$ & $16.8 \pm 3.5$ & -0.23 & $>0.05$ \\
\hline \%Power meal & $21.07 \pm 6.07$ & $23.5 \pm 6.5$ & -0.75 & $>0.05$ \\
\hline Power rest DF & $2417 \pm 801$ & $1209 \pm 251$ & 3.5 & $<0.01$ \\
\hline Power meal DF & $5215 \pm 2481$ & $4896 \pm 1541.5$ & 0.28 & $>0.05$ \\
\hline PR & $2.13 \pm 0.6$ & $4.3 \pm 1.8$ & -3.3 & $<0.01$ \\
\hline
\end{tabular}

$\mathrm{DF}=$ dominant frequency. $\mathrm{PR}=$ power ratio.

$\mathrm{P}<0.05=$ Significant. $\quad \mathrm{P}<0.01=$ highly significant.

There was no significant difference between male and female cases regarding EGG parameters $(\mathrm{P}>0.05)$.
While there was a statistically highly significant increase in the mean of power rest at $\mathrm{DF}(\mathrm{P}<0.05)$ and mean PR $(<0.01)$ in males compared to females in the control group.

Study of distribution of postprandial dip among different groups: is shown in table (8).

Table (8):Distribution of postprandial dip among different groups:

\begin{tabular}{|l|l|l|l|l|l|}
\hline Dip & $\begin{array}{l}\text { Group I } \\
\text { № \& \% }\end{array}$ & $\begin{array}{l}\text { Group II } \\
\text { № \& \% }\end{array}$ & $\begin{array}{l}\text { Group III } \\
\text { № \& \% }\end{array}$ & X2 & P \\
\hline Absent & $533.3 \%$ & $533.3 \%$ & $4 \quad 26.7 \%$ & \multirow{2}{*}{2.6} & $>0.05$ \\
\hline Present & $1066.7 \%$ & $1066.7 \%$ & $1173.3 \%$ & \\
\hline
\end{tabular}

$\mathrm{X} 2=$ Chi square. $\quad$ № $=$ Number.

$\%=$ Percentage of all. 


\section{El Bokl ,Mohammed, et al}

Where the postprandial dip was present $66.7 \%$ in group I, $80 \%$ in group
II and $93.3 \%$ among group III, with an insignificant difference $(\mathrm{P}>0.05)$.

Results of visual analysis of EGG and distribution of gastric dysrhythmia among the study groups are shown in table (9):

Table (9):Comparison between 3 groups regarding gastric dysrhythmia:

\begin{tabular}{|c|c|c|c|c|c|}
\hline Group & $\begin{array}{l}\text { Group I } \\
\text { № \&\%\% }\end{array}$ & $\begin{array}{l}\text { Group II } \\
\text { № \& \% } \%\end{array}$ & $\begin{array}{l}\text { Group III } \\
\text { № \& \% }\end{array}$ & $\mathrm{X} 2$ & $\mathrm{P}$ \\
\hline Normal & $10 \quad 66.7 \%$ & $1280 \%$ & $14 \quad 93.3 \%$ & \multirow{3}{*}{3.3} & \multirow{3}{*}{$>0.05$} \\
\hline Bradygastria & $16.7 \%$ & $320 \%$ & $3.3 \%$ & & \\
\hline Tachygastria & $\begin{array}{ll}4 & 26.7 \% \\
\end{array}$ & 0 & 0 & & \\
\hline
\end{tabular}

$\mathrm{X} 2=$ Chi square.

Normogastric subjects: Were 10 patients $(66.7 \%)$ in group I (without portal gastropathy), 12 patients $(80 \%)$ in group II (with portal gastropaty), and 14 subjects $(93.3 \%)$ in group III (controls). While bradygastric subjects: Were one
$(6.7 \%)$ in group I, 3 patients $(20 \%)$ in group II and one subject $(3.3 \%)$ in group III.

Tachygastria was present in 4 patients $(26.7 \%)$ in group I, and absent among groups II and III.

* Comparison of results of gastric emptying in different groups, was shown in table (10) :-

Table (10): Comparison between different groups as regards gastric emptying.

\begin{tabular}{|l|l|l|l|l|l|}
\hline G.emptying & Group I & Group II & Group III & $\mathrm{T}$ & $\mathrm{P}$ \\
\hline $1 / 2$ empt.time & $19 * \pm 7.38$ & $25.4 * \pm 14$ & $20.9 \pm 6.6$ & 2.3 & $<0.05$ \\
\hline Full empt.time & $47.9 \pm 10.3$ & $58.9 * \pm 13$ & $45 * \pm 13.5$ & 4.2 & $<0.0 .5$ \\
\hline Fasting antrum & $5.1 \pm 2.9$ & $4.8 \pm 1.4$ & $4.4 \pm 1.68$ & 1.2 & $>0.05$ \\
\hline Full ant. & $15.9 \pm 3.5$ & $12.7 \pm 3.9$ & $12.12 \pm 3.3$ & 1.6 & $>0.05$ \\
\hline
\end{tabular}

$1 / 2$ emp.t.=half emptying time(min).

Full emp.t.=full emptying time(min.).

Fasting antrum=area of fasting antrum $(\mathrm{Cm} 2)$.

Full antrum $=$ area of full antrum $(\mathrm{C} \mathrm{m} 2)$.

The mean of $1 / 2$ emptying time among group I was 19.06 min., 25.4 min. in group II and 20.9 min. in group III with a statistically significant increase in group II compared to group I $(\mathrm{P}<0.05)$.

The mean of full emptying time among group I was $47.9 \mathrm{~min} ., 58.9 \mathrm{~min}$. in group II and $45.2 \mathrm{~min}$. in group III, which has a statistically significant increase in group II compared to group III $(\mathrm{P}<0.05)$.

The mean of fasting antral area was $5.1 \mathrm{~cm} 2$ in group $\mathrm{I}, 4.8 \mathrm{~cm} 2$ in group II and $4.4 \mathrm{~cm} 2$ in group III with a st atistically insignificant difference ( $\mathrm{P}>0.05)$.

The mean of full antral area was $15.9 \mathrm{~cm} 2$ in group I, $12.7 \mathrm{~cm} 2$ in group II and $12.1 \mathrm{~cm} 2$ in group III with an insignificant difference $(\mathrm{P}>0.05)$. 
Correlation between results of gastric emptying and the grade of Child Pugh classification; is shown in table (11) :

Table (11): Comparison between grades of Child pugh classification regarding gastric emptying:

\begin{tabular}{|c|c|c|c|c|c|}
\hline G.emptying $\quad$ Grade & Child A & Child B & Child C & $\mathrm{t}$ & $\mathrm{P}$ \\
\hline 1/2emptying time & $20.07 \pm 6.8$ & $24 \pm 16.7$ & $24.3 \pm 12.07$ & 0.43 & $>0.05$ \\
\hline Full empt.time & $53.3 \pm 13.7$ & $54.6 \pm 13.7$ & $51.6 \pm 12.4$ & 0.09 & $>0.05$ \\
\hline Fasting antral area & $5 \pm 2.9$ & $5.3 \pm .164$ & $4.3 \pm 1.2$ & 0.35 & $>0.05$ \\
\hline Full anral area & $15.8 \pm 3.7$ & $12.96 \pm 3.8$ & $12.95 \pm 5.5$ & 2.05 & $>0.05$ \\
\hline
\end{tabular}

P> 0.05= insignificant.

We have studied the correlation

areas) and grade of Child Pugh between different gastric emptying parameters ( $1 / 2$ emptying time, full emptying time, fasting and full antral classification, and there was a statistically insignificant correlation between different groups.

Correlation between results of gastric emptying and the difference in gender in the studied groups, are shown in tables (12\&13):

Table (12): Effect of gender among cases on gastric emptying:

\begin{tabular}{|l|l|l|l|l|}
\hline G.emptying Gender & Male & Female & $\mathrm{t}$ & $\mathrm{P}$ \\
\hline $1 / 2$ emptying time & $22.5 \pm 11.7$ & $21 \pm 12.4$ & 0.28 & $>0.05$ \\
\hline Full emptying time & $52.3 \pm 13.5$ & $57.7 \pm 10.9$ & -0.89 & $>0.05$ \\
\hline Fasting antral area & $4.97 \pm 2.4$ & $4.96 \pm 1.4$ & 0.01 & $>0.05$ \\
\hline Full antral area & $14.5 \pm 4.4$ & $13.3 \pm 1.85$ & 0.66 & $>0.05$ \\
\hline
\end{tabular}

P> 0.05= insignificant.

Table (13): Effect of gender among controls on gastric emptying:

\begin{tabular}{|l|l|l|l|l|}
\hline G.emptying & Male & Female & $\mathrm{t}$ & $\mathrm{P}$ \\
\hline $1 / 2$ emptying time & $23.3 \pm 6.8$ & $17.2 \pm 4.9$ & 1.9 & $>0.05$ \\
\hline Full empt. time & $50.2 \pm 9.2$ & $37.7 \pm 16.3$ & 1.9 & $<0.05$ \\
\hline Fasting antral area & $4.6 \pm 1.7$ & $4.12 \pm 1.6$ & 0.55 & $>0.05$ \\
\hline Full antral area & $12.6 \pm 3.4$ & $11.4 \pm 3.3$ & 0.6 & $>0.05$ \\
\hline
\end{tabular}

$\mathrm{P}>0.05=$ insignificant. $\quad \mathrm{P}<0.05=$ significant.

There was statistically insignificant correlation between males and females as regards gastric emptying in group I and group II $(\mathrm{P}>0.05)$.
While there was a significant increase in full emptying time among males compared to females in group III $(\mathrm{P}<0.05)$. 
Correlation between EGG parameters and results of gastric emptying among the studied groups; as shown in tables $(14),(15),(16)$ :

Table (14): Correlation between EGG parameters and gastric emptying values among group I (r. test).

\begin{tabular}{|c|c|c|c|c|}
\hline EGG & $\begin{array}{l}\text { 1/2emp. } \\
\text { time }\end{array}$ & $\begin{array}{l}\text { Full } \\
\text { emp.t. }\end{array}$ & Fasting antrum & $\begin{array}{l}\text { Full } \\
\text { antrum }\end{array}$ \\
\hline$\%$ CPM rest & $\begin{array}{l}\text { r. }-0.09 \\
P>0.05\end{array}$ & $\begin{array}{l}\text { r. }-0.07 \\
\mathrm{P}>0.05\end{array}$ & $\begin{array}{l}\text { r. } 0.11 \\
P>0.05\end{array}$ & $\begin{array}{l}\text { r. } 0.15 \\
\mathrm{P}>0.05\end{array}$ \\
\hline$\% \mathrm{CPM}$ meal & $\begin{array}{l}\text { r. }-0.18 \\
P>0.05\end{array}$ & $\begin{array}{l}\text { r. }-0.007 \\
P>0.05\end{array}$ & $\begin{array}{l}\text { r. } 0.07 \\
P>0.05\end{array}$ & $\begin{array}{l}\text { r. } 0.47 \\
P>0.05\end{array}$ \\
\hline DF & $\begin{array}{l}\text { r. } 0.15 \\
P>0.05\end{array}$ & $\begin{array}{l}\text { r. } 0.025 \\
P>0.05\end{array}$ & $\begin{array}{l}\text { r. } 0.007 \\
P>0.05\end{array}$ & $\begin{array}{l}\text { r. }-0.06 \\
P>0.05\end{array}$ \\
\hline$\%$ Power rest & $\begin{array}{l}\text { r. } 0.34 \\
P>0.05\end{array}$ & $\begin{array}{l}\text { r. } 0.19 \\
P>0.05\end{array}$ & $\begin{array}{l}\text { r. } 0.52 \\
P>0.05\end{array}$ & $\begin{array}{l}\text { r. } 0.56 \\
P>0.05\end{array}$ \\
\hline$\%$ Power meal & $\begin{array}{l}\text { r. } 0.46 \\
P>0.05\end{array}$ & $\begin{array}{l}\text { r. } 0.46 \\
P>0.05\end{array}$ & $\begin{array}{l}\text { r. } 0.03 \\
>0.05\end{array}$ & $\begin{array}{l}\text { r. } 0.09 \\
P>0.05\end{array}$ \\
\hline Power rest DF & $\begin{array}{l}\text { r. } 0.11 \\
P>0.05\end{array}$ & $\begin{array}{l}\text { r. } 0.43 \\
P>0.05\end{array}$ & $\begin{array}{l}\text { r. }-0.04 \\
P>0.05\end{array}$ & $\begin{array}{l}\text { r. } 0.0003 \\
P>0.05\end{array}$ \\
\hline Power meal DF & $\begin{array}{l}\text { r. } 0.23 \\
\mathrm{P}>0.05\end{array}$ & $\begin{array}{l}\text { r. } 0.12 \\
P>0.05\end{array}$ & $\begin{array}{l}\text { r. } 0.03 \\
P>0.05\end{array}$ & $\begin{array}{l}\text { r. } 0.14 \\
P>0.05\end{array}$ \\
\hline PR & $\begin{array}{l}\text { r. }-0.26 \\
P>0.05\end{array}$ & $\begin{array}{l}\text { r. }-0.59 * \\
\mathrm{P}<0.05\end{array}$ & $\begin{array}{l}\text { r. } 0.55 \\
P>0.05\end{array}$ & $\begin{array}{l}\text { r. }-0.29 \\
P>0.05\end{array}$ \\
\hline
\end{tabular}

$\mathrm{P}>0.05=$ insignificant.

$\mathrm{P}<0.05=$ significant.

$*=$ the significant correlation.

Table (15): Correlation between EGG parameters and gastric emptying values among group II.

\begin{tabular}{|c|c|c|c|c|}
\hline EGG & 1/2emp.t. & Full emp.t. & Fasting antrum & Full antrum \\
\hline$\% \mathrm{CPM}$ rest & $\begin{array}{l}\text { r. }-0.2 \\
P>0.05\end{array}$ & $\begin{array}{l}\text { r. } 0.35 \\
P>0.05\end{array}$ & $\begin{array}{l}\text { r. }-0.4 \\
P>0.05\end{array}$ & $\begin{array}{l}\text { r. } 0.4 \\
P>0.05\end{array}$ \\
\hline$\% \mathrm{CPM}$ meal & $\begin{array}{l}\text { r. } 0.42 \\
P>0.05\end{array}$ & $\begin{array}{l}\text { r. } 0.11 \\
P>0.05\end{array}$ & $\begin{array}{l}\text { r. }-0.27 \\
P>0.05\end{array}$ & $\begin{array}{l}\text { r. } 0.29 \\
P>0.05\end{array}$ \\
\hline DF & $\begin{array}{l}\text { r. }-0.47 \\
\mathrm{P}>0.05\end{array}$ & $\begin{array}{l}\text { r. } 0.08 \\
P>0.05\end{array}$ & $\begin{array}{l}\text { r. }-0.38 \\
P>0.05\end{array}$ & $\begin{array}{l}\text { r. } 0.4 \\
P>0.05\end{array}$ \\
\hline \% Power rest & $\begin{array}{l}\text { r. } 0.4 \\
P>0.05\end{array}$ & $\begin{array}{l}\text { r. } 0.3 \\
P>0.05\end{array}$ & $\begin{array}{l}\text { r. } 0.3 \\
P>0.05\end{array}$ & $\begin{array}{l}\text { r. } 0.29 \\
P>0.05\end{array}$ \\
\hline$\%$ Power meal & $\begin{array}{l}\text { r. } 0.09 \\
P>0.05\end{array}$ & $\begin{array}{l}\text { r. } 0.31 \\
P>0.05\end{array}$ & $\begin{array}{l}\text { r. }-0.46 \\
P>0.05\end{array}$ & $\begin{array}{l}\text { r. } 0.57 * \\
\mathrm{P}<0.05\end{array}$ \\
\hline Power rest DF & $\begin{array}{l}\text { r. } 0.49 * \\
\mathrm{P}<0.05\end{array}$ & $\begin{array}{l}\text { r. }-0.07 \\
P>0.05\end{array}$ & $\begin{array}{l}\text { r. } 0.53 \\
P>0.05\end{array}$ & $\begin{array}{l}\text { r. }-0.19 \\
P>0.05\end{array}$ \\
\hline Power meal DF & $\begin{array}{l}\text { r. } 0.7 \quad * \\
\mathrm{P}<0.05\end{array}$ & $\begin{array}{l}\text { r. } 0.2 \\
P>0.05\end{array}$ & $\begin{array}{l}\text { r. } 0.42 \\
P>0.05\end{array}$ & $\begin{array}{l}\text { r. } 0.05 \\
P>0.05\end{array}$ \\
\hline PR & $\begin{array}{l}\text { r. } 0.29 \\
P>0.05\end{array}$ & $\begin{array}{l}\text { r. } 0.52 \\
\mathrm{P}<0.05\end{array}$ & $\begin{array}{l}\text { r. }-0.2 \\
P>0.05\end{array}$ & $\begin{array}{l}\text { r. } 0.36 \\
P>0.05\end{array}$ \\
\hline
\end{tabular}

$\mathrm{P}>0.05=$ insignificant.

$\mathrm{P}<0.05=$ significant

$*=$ significant correlation. 
Table (16) Correlation between EGG parameters and gastric emptying values among group III.

\begin{tabular}{|l|l|l|l|l|}
\hline EGG & $\begin{array}{l}1 / 2 \text { Gempt. } \\
\text { time }\end{array}$ & $\begin{array}{l}\text { Full } \\
\text { emp.t. }\end{array}$ & Fasting antrum & $\begin{array}{l}\text { Full } \\
\text { antrum }\end{array}$ \\
\hline \%CPM rest & $\begin{array}{l}\text { r. } 0.072 \\
\text { P }>0.05\end{array}$ & $\begin{array}{l}\text { r. } 0.26 \\
\text { P }>0.05\end{array}$ & $\begin{array}{l}\text { r. } 0.075 \\
\text { P }>0.05\end{array}$ & $\begin{array}{l}\text { r. } 0.06 \\
\text { P }>0.05\end{array}$ \\
\hline \%CPM meal & r. -0.09 & r. 0.1 & r. 0.07 & r. 0.07 \\
& P $>0.05$ & P $>0.05$ & P $>0.05$ & P $>0.05$ \\
\hline \multirow{2}{*}{$\%$ Power rest } & r. -0.1 & r. 0.08 & r. -0.09 & r. -0.03 \\
& P $>0.05$ & P $>0.05$ & P $>0.05$ & P $>0.05$ \\
\hline \multirow{2}{*}{$\%$ Power meal } & r. 0.07 & r. -0.006 & r. 0.04 & r. -0.03 \\
& P $>0.05$ & P $>0.05$ & P $>0.05$ & P $>0.05$ \\
\hline \multirow{2}{*}{ Power rest DF } & r. 0.37 & r. 0.3 & r. 0.25 & r. -0.12 \\
& P $>0.05$ & P $>0.05$ & P $>0.05$ & P $>0.05$ \\
\hline Power meal & r. 0.08 & r. 0.004 & r. 0.26 & r. -0.07 \\
DF & P $>0.05$ & P $>0.05$ & P $>0.05$ & P $>0.05$ \\
\hline \multirow{2}{*}{ PR } & r. -0.22 & r. -0.26 & r. -0.17 & r. -0.16 \\
& P $>0.05$ & P $>0.05$ & P $>0.05$ & P $>0.05$ \\
\hline
\end{tabular}

$\mathrm{P}>0.05=$ insignificant.

Shows a significant indirect correlation only between PR and full emptying time $(\mathrm{P}>0.05)$ in patients without portal hypertensive gastropathy (group I).

And shows a significant direct correlation between $\%$ power meal and area of full antrum in group II $(\mathrm{P}<0.05)$, a significant direct correlation between power rest at DF and $1 / 2$ emptying time in group II $(\mathrm{P}<0.05)$, a significant direct correlation between power meal at DF and $1 / 2$ emptying time, and a significant direct correlation between PR and full emptying time in group II $(\mathrm{P}<0.05)$.

And shows a statistically insignificant correlation between EGG and results of gastric emptying in controls (group III) ( $\mathrm{P}>0.05)$.

\section{Discussion}

Liver cirrhosis is a common disease all over the world with its different etiologies. Investigations for motility disorders may explain dyspeptic symptoms encountered in liver cirrhosis (Barnert et al., 1997).
Gastric emptying, an important parameter of gastric motility, shows a considerable heterogenicity in liver cirrhosis. Faster gastric emptying in human liver cirrhosis and in portal hypertension may be possibly due to reduced gastric wall compliance, with a consequent increase in intragastric pressure. Delayed gastric emptying of liquid components of a meal has also been noted in patients with liver cirrhosis. The mechanism responsible for abnormal gastric motility in liver cirrhosis is not well understood, but microcirculation in gastric walls, neural and hormonal factors may be essential to the regulation of gastric emptying (Usami et al., 1998). Gastric motility is controlled by gastrointestinal hormones (e.g. gastrin, secretin, motilin, cholecystokinin and glucagon) and innervated by sympathetic and parasympathetic nerves, as well as by the mural and myenteric nerve plexus. Abnormalities in neural control and gastrointestinal hormones may be the 


\section{El Bokl ,Mohammed, et al}

ause in cases of liver cirrhosis (Isobe et al., 1994).

In this study we found that $70 \%$ of patients were complaining of dyspeptic symptoms (such as; nausea, vomiting, epigastric pain, fullness, and bloating). All the patients with portal hypertensive gastropathy (group II) were complaining of such symptoms. While six patients $(40 \%)$ without portal hypertensive gastropathy (group I) were complaining of some of these symptoms.

On studying the correlation between dyspeptic symptoms and EGG variables, it was found that there was no significant correlation between symptoms and EGG parameters.

This agree with the results obtained by Jebbink et al., 1995 who found no significant correlation between any of EGG variables and symptoms. Regarding the study of EGG pattern in preprandial and postprandial periods in different groups; the study showed that the percentage of normal dominant frequency at fasting and postprandial period. in cirrhotic patients (with or without portal hypertensive gastropathy) showed a decrease in the percentage of normal dominant frequency at fasting and postprandial periods, compared to the control group, although the difference was statistically insignificant $(\mathrm{P}>0.05)$.: The mean of group I was 69.4 in fasting and 68.8 in postprandial state, the mean of group II was 66.7 in fasting and 63.4 in the postprandial state, and it was in group III 73.9 in fasting and 73.8 postprandially

On studying the percentage of power at DF in fasting state patients with liver cirrhosis without portal hypertensive gastropathy (group I) showed insignificant increase compared to patients with portal hypertensive gastropathy (group II) and control group $(\mathrm{P}>0.05)$ (The mean of group I was 19.65, group II was 16.7 , and in group III was 16.6, table 4).

Also (group I) showed statistically insignificant increase in the percentage of power at DF in the postprandial state compared to group II and III $(\mathrm{P}>0.05)$ (the mean in group I was 23.6, in group II was 23.5, and in group III was 22.06, table 4).

Studying the power at rest of DF showed a highly significant increase in group I compared to group II and III $(\mathrm{P}<0.01)$, (the mean of group I was 2944.9 , in group II was 2477 , and was 1934 in group III, table 4).

While the power meal at DF was higher in group I than in group II and III, however, without statistical signific -ance, the mean in group I was 5922.5, in group II was 4804.8, and was 5087.8 in group III $(\mathrm{P}>0.05)$.

These results didn't correlate with that obtained by (Miyajima et al., 2001) who studied 27 patients with liver cirrhosis symptoms, haematological, . By using ambulatory EGG recorder, EGG was obtained at rest and continuously recorded $2 \mathrm{~h}$ after a meal. By using FFT (Fast Fourier Transform) analysis, DF (dominant frequency) and their amplitudes were obtained from 4channel EGG during fasting and after a meal. They found less values in cirrhotic patients than controls. The mean of the peak power obtained in cirrhotic patients during fasting was 126 Vpp and $97 \mathrm{Vpp}$ after the meal. The mean of the peak power obtained in controls during fasting was $238 \mathrm{Vpp}$ and $327 \mathrm{Vpp}$ after the meal.

Regarding the power ratio (PR), it was lower in patients with cirrhosis (group I and II) compared to controls (group III). However, this was not statistically significant $(\mathrm{P}>0.05)$. The 
mean in group I was 2.01, in group II was 1.93 , and was 2.63 in group III (Table 4).

This coincides with the results of (Miajima et al., 2001), who found that the power ratio (PR) was higher among healthy controls (1.37) than that of cirrhotic patients (0.77). This also coincides with the results obtained by (Usami et al., 1998), they studied 36 patients with liver cirrhosis. EGG and gastric emptying using radioactive technique were done. They found less PR (power ratio)in cirrhotic patients than controls, thus, demonstrating dysrhythmic EGG waveforms for liver cirrhosis.

Results of EGG parameters in different grades of Child Pugh classification showed statistically insignificant increase in the power ratio in Child B group compared to Child A and $\mathrm{C}(\mathrm{P}>0.05)$.

While, \%CPM was significantly higher in Child A group of patients than group $\mathrm{B}$ and $\mathrm{C}$.

These results didn't correlate with the results of (Miajima et al., 2001) who found significant increase in the power ratio in Child $\mathrm{A}$ and controls compared to Child B and C.

Also, (Miajima et al., 2001) found no significant difference in the frequency ratio among the different grades of Child classification.

The distribution of the postprandial dip was statistically insignificant, however the lower percentage of the presence of the postprandial dip was among cirrhotic patients (with or without portal hypertensive gastropathy) compared to group III (controls) (table 8).

During the study of gastric dysrhythmia by visual analysis of EGG, we have found that the lowest percentage of normogastria was in patients without portal hypertensive gastropathy (66.7\%), while was $80 \%$ in patients with portal hypertensive gastropathy, and $93.3 \%$ in controls. The higher percentage of bradygastria was present among patients with portal hypertensive gastropathy (group II) reaching $20 \%$, compared to $6.7 \%$ in patients without portal hypertensive gastropathy (group I) and $3.3 \%$ in controls.

While tachygastria was present in high percentage in group I reaching $26.7 \%$ and no tachygastric subjects among group II and III (Table 9).

We have found a significant delay in gastric emptying in cirrhotic patients with portal hypertensive gastropathy (group II) compared to patients without PHG (group I) and control group; the $1 / 2$ emptying and full emptying times were significantly increased in group II patients versus group I and III $(\mathrm{P}<0.05)$ (Table 11).

On the other hand, the fasting antral area and the postprandial antral area were increased in group I than group II and III (Table 10).

These results agree with (Usami et al., 1998), who found that $1 / 2$ emptying time was significantly prolo nged in cirrhotic patients compared to controls.

Also, these results correlated with what obtained by (Barnert et al., 1997), who studied 15 patients with liver cirrhosis who had no portal hypertensive gastropathy or antral ectasia. An ultrasonographic method was used for estimation of gastric emptying of liquid low caloric meal and of a semisolid meal richer in calories. They found insignificant increase in $1 / 2$ emptying time in cirrhotic patients (without portal gastropathy) compared to controls.

They also found that, fasting and postprandial antral areas were the same among cirrhotic patients and controls. 


\section{El Bokl ,Mohammed, et al}

On the other hand (Barnert et al., 1997), studied gastric emptying with two types of test meals in patients with liver cirrhosis. They reported an accelerated rate of gastric emptying of a liquid low caloric meal in cirrhotics and of a near normal gastric emptying of a semisolid meal with a higher caloric load.

On studying gastric emptying in different grades of Child Pugh classification; nave found that the full emptying time was higher in Child B group compared to Child A and C, however, statistically insignificant $(\mathrm{P}>0.05)$ (Table 12).

This coincides with the results of (Acalovschi et al., 1997), who found increase in the full emptying time in Child B patients, indicating impaired gastric emptying.

So, in this study we have found a delayed gastric emptying in cirrhotic patients with portal hypertensive gastropathy in comparison to those without portal hypertensive gastropathy and controls. This signifies the importance of portal hypertensive gastropathy in disturbed gastric motility.

We have studied the correlation between EGG variables and parameters of gastric emptying in group I (without portal hypertensive gastropathy), and we have found statistically significant indirect correlation between PR and full emptying time $(\mathrm{P}<0.05) \quad($ table 15), denoting that increase in $P R$ is accompanied by decrease in full emptying time, or in other words faster gastric emptying.

Previous investigations of gastric emptying in liver cirrhosis gave very controversial results. Some authors described prolonged gastric emptying in cirrhosis. Chesta et al., 1990, using a solid meal, found delayed gastric emptying; Isobe et al. In 1994 also found delayed gastric emptying in cirrhosis, using a radioisotopic method and a semisolid meal. Other studies reported accelerated gastric emptying in portal hypertensive in humans with presinusoidal portal hypertension. Congestion of the gastric wall probably brings about altered compliance of the stomach. This hypothesis is supported by the fact that acceleration of gastric emptying occurs with liquids, whose emptying is controlled by fundic compliance responses.

So, it seems that these controversial results regarding gastric emptying in cirrhotic patients may be explained by the fact that most of the studies compared cirrhotic patients as one group to healthy subjects. But on studying cirrhotic patients with and without portal hypertensive gastropathy, as we did in our study, we could reach to more accurate results. It is expected that portal hypertensive gastropathy with congestion of the gastric wall, probably brings about altered compliance of the stomach, resulting in the altered motility.

\section{Conclusion :-}

In this study it was concluded that there was disturbance in gastric motility in cirrhotic patients in form of changes in EGG recording results \& ultrasonic gastric emptying results this distutbbance were more evident in cerrhotic patients with portal hyperte nsive gastropathey which may point to the effect of PHG on the gastric wall compliance \& may reflect the idea of treatment of $\mathrm{PHG}$ on normalization effectiveness of gastric dysmotility EGG pattern, in the future.

\section{Reeferences}

1. Acalovschi,M.:Dumetrascud,D.L. and Caskany,I. (1997) :Gastric and gall bladder emptying of a mixed meal not coordinated in liver 
cirrhosis; a simultaneous sonografic study. Gut, 40:412.

2. Agnihotri, N.; Kaur, S.; Bahadur, J. et al. (1996): Diminution in parietal cell number in experimental portal hypertensive gastropathy. Dig. Dis. Sci., 42:431.

3. Balan, K.K.; Grime, S.; Sutton, R. et al. (1996): Abnormalities of gastric emptying in portal hypertension. Am. J. Gastroenterol., 91(3): 530.

4. Barnert, J.; Dumitrascu, D.L. and Wienbeck, M. (1997): Gastric emptying in liver cirrhosis. The effect of the type of meal. Europ. J. Gastroenerol. Hepatol., 9: 1073.

5. Bernard, B.; Cardanel, J.F.; Valla,D. et al.(1995): Prognostic significance of bacterial infection in cirrhotic patients: A prospective study. Gastroentrol.,108:1824.

6. Chen, J. and McCallum, R.W. (1991): Response of electric activity in human stomach to water and a solid meal. Med. Biol. Eng. Comput., 29:351.

7. Chen, J. and McCallum, R.W. (1992): gastric slow wave abnormalities in patients with gastroparesis. Am. J. Gastroentrol.,87: 477.

8. Chen, J.D. and McCallum, R.W. (1993): Clinical applications of electrogastrography. Am. J. Gastroentrol., 88:1324.

9. Chen, J.; Richard, R.D. and McCallum, R.W. (1993): Frequency components of EGG and their correlations with gastrointestinal motility. Med. Biol. Eng. Comput., 31:60.

10. Chen, J.D.; Co, E.; Lain, J. and Pan, J. (1997): Pattern of myoelectrical activity in human subjects of different ages. Am. J. Physiol., 273(5): 1022.
11. Chesta,J.,Lilo,R.; Defillippi, Cet al. (1991): Orocecal transit time and gastric emptying in patients with cirrhosis. Gastroenterol., 36:917

12. Gostout,C.L.(1992) The clinical endoscopic spectrum of watemelon stomach.J. clin Gastroenterol., $15: 256$

13. Grassi, M.; Albiani, B.; De Matteis, A. et al. (2001): Prevalence of dyspepsia in liver cirrhosis. A clinical and epidemiological investigation. Minerva Med., 92(1): 7.

14. Groszmann, R.J. and Colombato, L.A. (1988): Gastric vascular changes in portal hypertension. Hepatology, 8:1708.

15. Groszmann, R.J. and Colombato, L.A. (1988): Gastric vascular changes in portal hypertension. Hepatology, 8:1708.

16. Hashizume, M.and Sugimachi,K. (1995) : classification of gastric lesions associated with portal hypertensionJ. Gastroenterol. Hepatol., 18:1139

17. Isobe, H.; Sakai, H.; Satoh, M. et al. (1994): Delayed gastric emptying in patients with liver cirrhosis. Dig. Dis. Sci., 39: 983.

18. Jebbink, H.L; Bruijs, PP.,Bravenber, B.et al.(1994): Gastric myoelectrical activity in patients with type I diabetes mellitus and autonomic neuropathy. Dig. Dis. Sci., Nov.;39(11).

19. Jebbink, H.J.A.; Van Berge, G.P.; Bruijs, P.P.M. et al. (1995): Gastric myoelectrical activity and gastrointestinal motility in patients with functional dyspepsia. Europ. J. Clin. Invest., 25: 429.

20. Johnson, L.R. (1992): Gastrointestinal physiology. In: Essential Medical Physiology. Byrne, J.H.; Downey, J.M. and 
Goodman, H.M. (eds). Raven Press. pp: 467.

21. Josep, M. and Pique', J.M. (1997): Portal hypertensive gastropathy. Balliere's Clin Gastroentrol. Vol 2. Josep, M. and Pique', J.M.(eds).Gastroentrol department. University of Barcelone, Villarroe. Pp:257.

22. Leone, O.; Zanelli, M.; Piana, S. et al. (1997): Late stage congestive portal gastropathy. J. Clin. Pathol., 50:350.

23. Lindberg, G.; Lwezon, M. and Hamad, B. (1996): 24 hour ambulatory EGG in healthy volunteers. Scand. J. Gastroentrol., 31(7):658.

24. Maijima ,H.,Noumura, M.; Muguruma, N.et al (2001); Relationship among gastric motility , autonomic activity ,and portal haemodynamics in patients with liver cirrhosis .j. gastroenterol. ,hepatol., 16:647.

25. Nishida, H.; Kodama, T.; Satoh, T. et al. (1989): $C$ hanges in gastric mucosal microcirculaton and mucosal lesions in portal hypertensive rats. J. Gastroentrol. Hepatol. 4 (suppl 1): 88.

26. Parkman, H.P.; Harris, H.D. and Urbain C. (1995): Gastroduodenal motility and dysmotility: An update on techniques available for evaluation. Am. J. Gastroentrol., 90(6):869.

27. Parkman, H.P.; Miller, M.A.; Trate, D. and Fisher, R. (1997):
Gastric emptying scintigraphy are complementary for assessment of dyspepsia. J. Clin. Gastroenterol., 24(44): 214.

28. Payen, J.L.; Cales,P.Viogt, J.et al.(1995): Sever portal Hypertensive gastropathy and antral vascular ectasia are distinct entities in patients with cirrhosis Gastroenterol, 108:138..

29. Sarin, S.K; Sreenivas, D.V.; Lahoti, D. et al. (1992): Factors influencing development of portal hypertensive gastropathy in patients with portal hypertension. Gastroenterol., 102: 994.

30. Smart, H.L and Triger, D.R. (1991): Clinical features, pathophysiology and relevance of portal hypertensive gastropathy. Endoscopy, 23: 224.

31. Smout, A.J.P.M.; Van Der Schee, E.J. and Grashuis, J.L. (1980): What is measured in electrogastrography? Dig. Dis. Sci., 25: 179 .

32. Usami, A.; Mizukami, Y. and Onji, M. (1998): Abnormal gastric motility in liver cirrhosis. Roles of secretin. Dig. Dis. Sci., 43: 2392.

33. Van Der Schee, E.J.; Smout, A.J.P.M. and Grashuis, J.L. (1982): Application of running spectrum analysis electrogastrog raphic signals recorded from dog and man. In: Weibeck, M. (ed.). Motility of digestive tract. Raven Press. New York. Pp59. 


\section{تأثير اعتلال المعدة الاحتقانى الناتج عن ارتفاع الضغط في الدورة الثئي

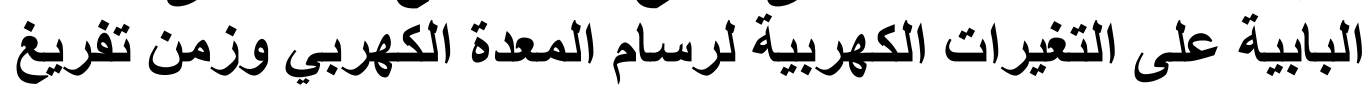

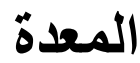

ا.د/ محمد البكل* \& د/ حنان محمود محمد بدوىه دم/ خالد حمدى عبد لمجيد

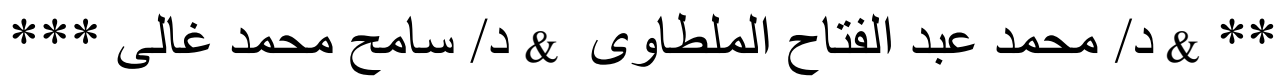
( أستاذ الإمر اض الباطنة** مدرس الإمر اض الباطنة ** \& \& أستاذ مساعد الإمر اض الباطنة****) بطب عين شمس الإطر

ان مـرض تليف الكبـد ، مـع تعـد أسبابه ، مـن الإمـر اض الثـــائعة بـين

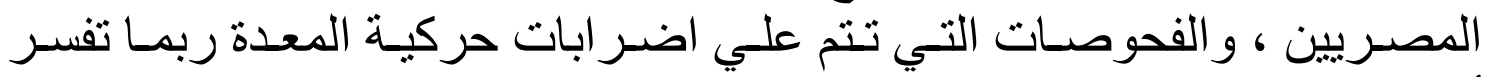

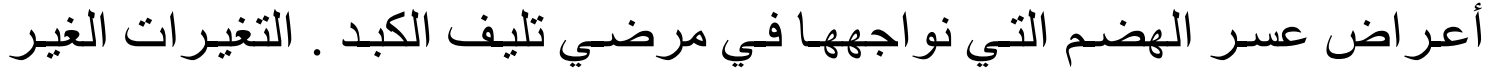

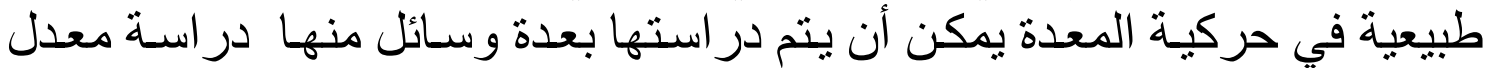

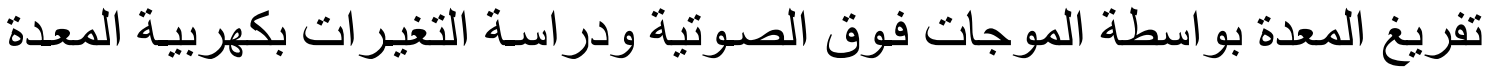

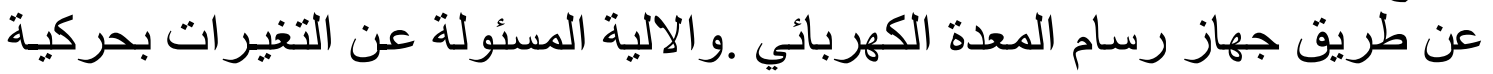

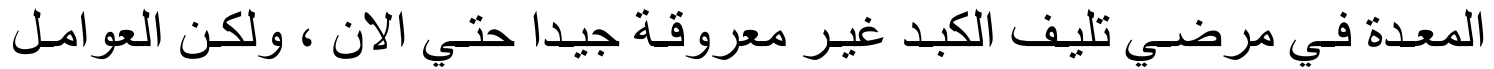

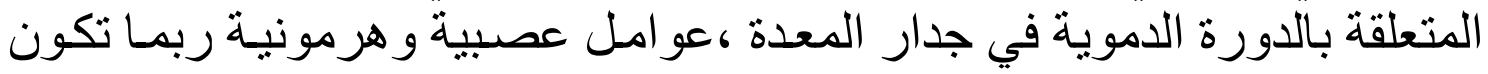

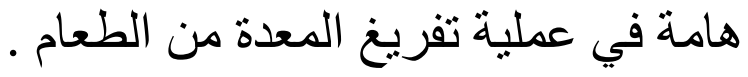

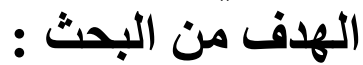
در اسة التغير ات بكهربية المعدة و معدل تفريغ المعدة عن طريق الموجيات

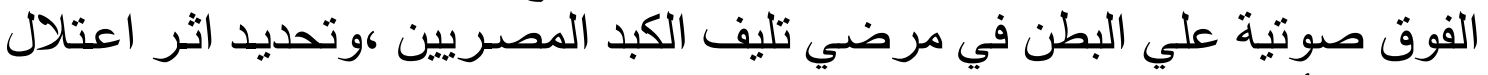

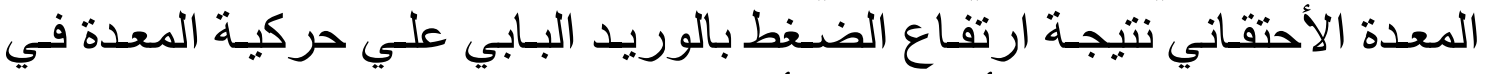

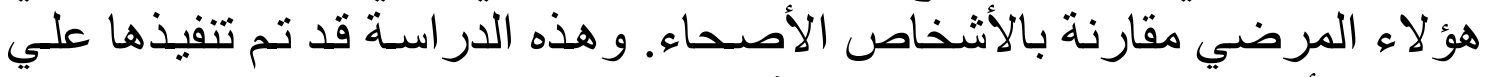

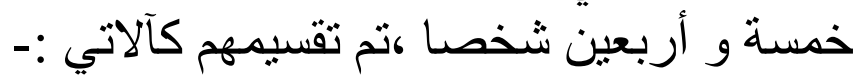

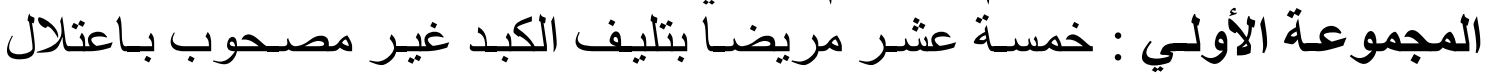

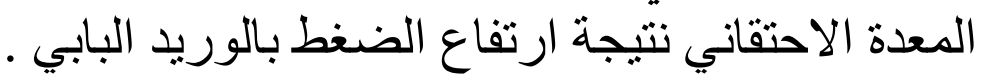

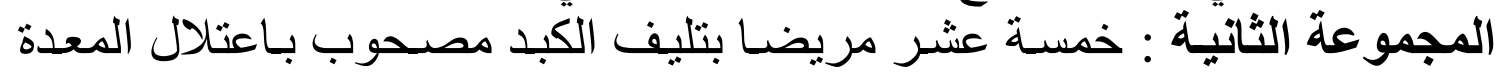

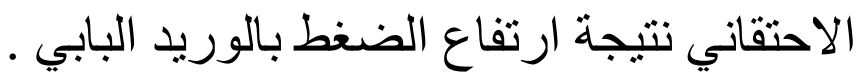

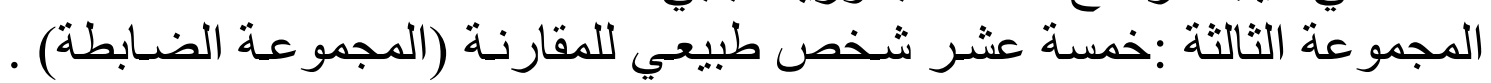
و الثلاث مجمو عات قد تم إخضاعهم للآتي :

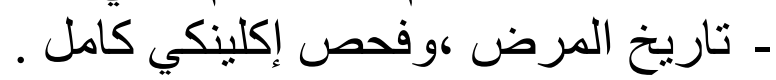

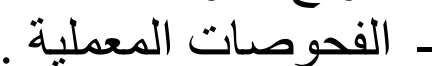

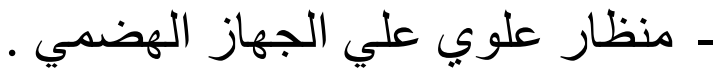
- رسم كهربائية المعدة في حالة الصيام وبعد تناول الطعام . 
ـ ـ أشعة بالموجات الفوق صـوتية علي البطن صـائما وبعد إعطاء وجبـة ثابتة لدر اسة معدل تفريغ المعدة .

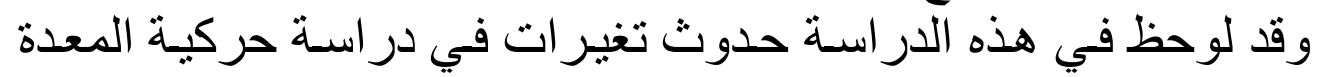

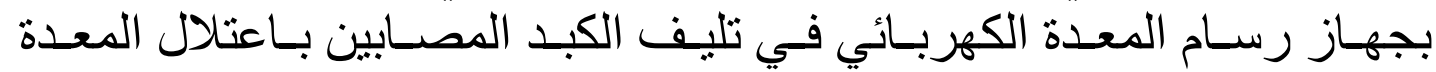

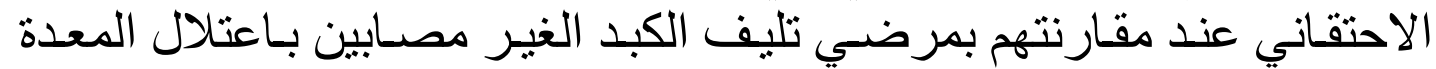

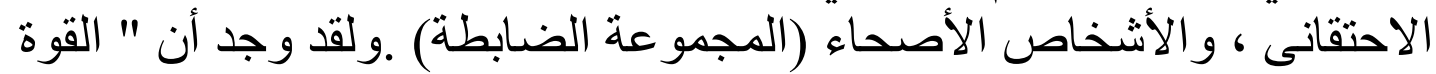

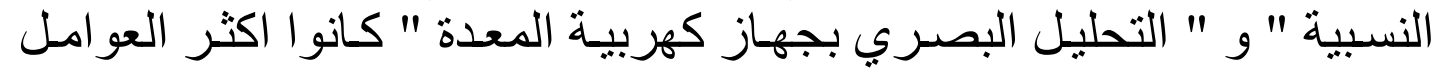

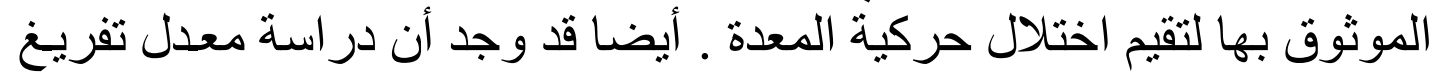

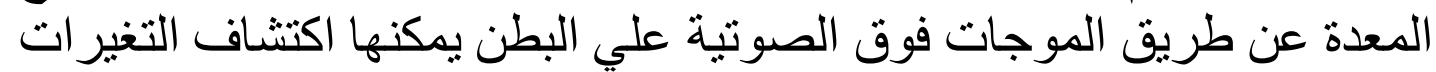

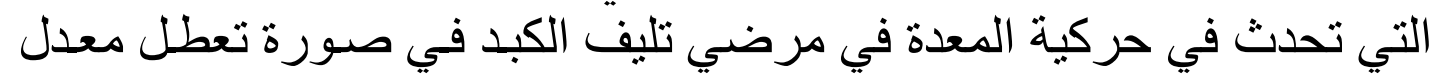

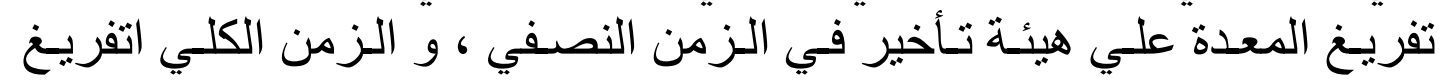

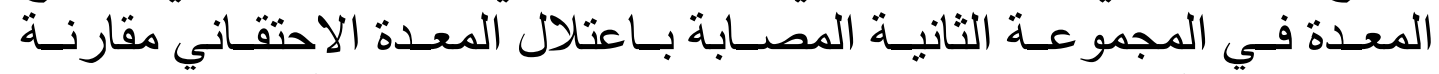

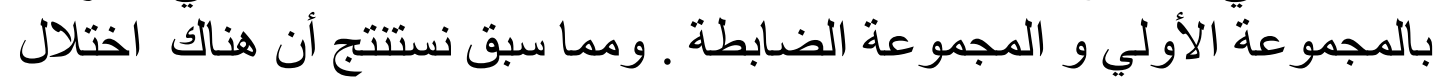

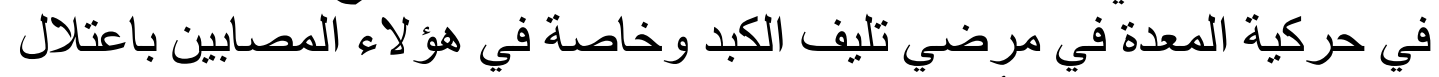

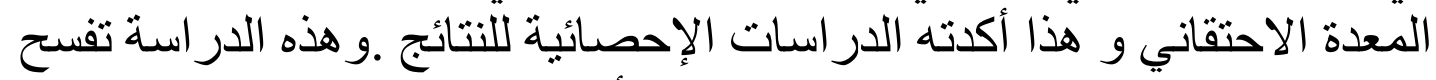

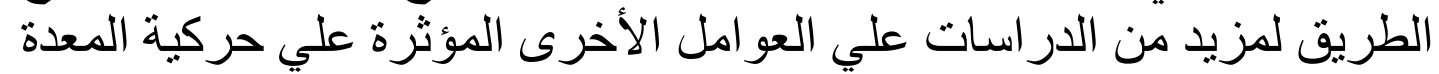

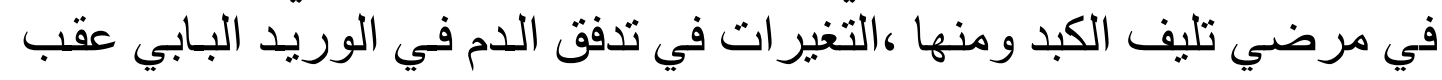

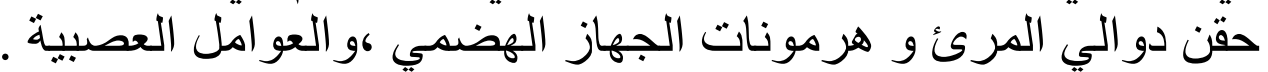

